\title{
Electronic Spectra of the Tetraphenylcyclobutadienecyclopentadienylnickel(II) Cation and Radical
}

\author{
P.R. Craig \\ Submitted to Journal of Physical Chemistry A
}

June 2016

Chemistry Department

Brookhaven National Laboratory

\author{
U.S. Department of Energy \\ [DOE Office of Science]
}

\begin{abstract}
Notice: This manuscript has been authored by employees of Brookhaven Science Associates, LLC under Contract No. DE- SC0012704 with the U.S. Department of Energy. The publisher by accepting the manuscript for publication acknowledges that the United States Government retains a non-exclusive, paid-up, irrevocable, world-wide license to publish or reproduce the published form of this manuscript, or allow others to do so, for United States Government purposes.
\end{abstract}




\section{DISCLAIMER}

This report was prepared as an account of work sponsored by an agency of the United States Government. Neither the United States Government nor any agency thereof, nor any of their employees, nor any of their contractors, subcontractors, or their employees, makes any warranty, express or implied, or assumes any legal liability or responsibility for the accuracy, completeness, or any third party's use or the results of such use of any information, apparatus, product, or process disclosed, or represents that its use would not infringe privately owned rights. Reference herein to any specific commercial product, process, or service by trade name, trademark, manufacturer, or otherwise, does not necessarily constitute or imply its endorsement, recommendation, or favoring by the United States Government or any agency thereof or its contractors or subcontractors. The views and opinions of authors expressed herein do not necessarily state or reflect those of the United States Government or any agency thereof. 


\section{Electronic Spectra of the Tetraphenylcyclobutadienecyclopentadienylnickel(II) Cation and Radical}

Peter R. Craig, ${ }^{\S}$ Zdeněk Havlas, ${ }^{\dagger}$ Marianela Trujillo, ${ }^{\S}$ Pawel Rempala, ${ }^{\S}$ James P. Kirby, ${ }^{\circledR}$ John R. Miller, ${ }^{\circledR}$ Bruce C. Noll, ${ }^{\S}$ and Josef Michl ${ }^{\dagger, \$ *}$

$\S$ Department of Chemistry and Biochemistry, University of Colorado, Boulder, CO 80309-0215, U.S.A., 'ं Institute of Organic Chemistry and Biochemistry, Academy of Science of the Czech Republic, 16610 Prague 6, Czech Republic, and "Chemistry Department, Brookhaven National Laboratory, Bldg. 555A, Upton, NY 11973, U.S.A. 
Abstract. Properties of the tetraphenylcyclobutadienecyclopentadienylnickel(II) cation $\mathbf{1}$ and its tetra-o-fluoro derivative 1a have been measured and calculated. The B3LYP/TZP optimized geometry of the free cation 1 agrees with a single-crystal X-ray diffraction structure except that in the crystal one of the phenyl substituents is strongly twisted to permit a close-packing interaction of two of its hydrogens with a nearby $\mathrm{BF}_{4}{ }^{-}$anion. The low-energy parts of the solution electronic absorption and magnetic circular dichroism (MCD) spectra of $\mathbf{1}$ and $\mathbf{1 a}$ have been interpreted by comparison with TD-DFT (B3LYP/TZP) results. Reduction or pulse radiolysis lead to a neutral 19electron radical, whose visible absorption and MCD spectra have been recorded and interpreted as well. The reduction is facilitated by $\sim 0.1 \mathrm{~V}$ upon going from 1 to $1 \mathbf{a}$. Unsuccessful attempts to prepare several other aryl substituted derivatives of $\mathbf{1}$ by the classical synthetic route are described in the Supporting Information.

$\S$ University of Colorado. † Institute of Chemistry and Biochemistry. I Brookhaven National Laboratory. 


\section{Introduction}

Transition metal complexes of tetraarylcyclobutadienes represent an interesting family of compounds that has been known for a long time, ${ }^{1}$ but whose electronic spectra have attracted attention only recently. The present paper deals with the electronic structure of the easily reducible closed-shell tetraphenylcyclobutadienecyclopentadienylnickel(II) cation $\mathbf{1}$ and its relation to that of the isoelectronic easily oxidizable uncharged tetraphenylcyclobutadienecyclopentadienylcobalt(I) sandwich complex 2, which we examined earlier (Scheme 1). ${ }^{2,3,4}$ Together, the Ni and Co metal complexes represent a complementary pair of redox centers whose four attachment points on the cyclobutadiene deck and a fifth set of attachment points on the cyclopentadiene deck provide ample opportunities for the preparation of more complicated constructs for molecular electronics, where the metal centers could serve as redox-sensitive gates.

We prepared a sample of $\mathbf{1}$ by a published procedure $^{5}$ from a dimeric tetraarylcyclobutenylpalladium(II) ${ }^{6}$ precursor. As described in the Supporting Information, we attempted to prepare other derivatives of $\mathbf{1}$ in the same fashion, but found that this synthetic route is not general. We succeeded in only one case, the tetrafluoro compound $\mathbf{1 a}$.

We have obtained the single-crystal X-ray structure of 1 , examined the electronic spectra of $\mathbf{1}$ and $\mathbf{1 a}$ in some detail using magnetic circular dichroism and time-dependent density functional theory, and compared their redox properties and the spectra of the corresponding neutral 19-electron radicals. 


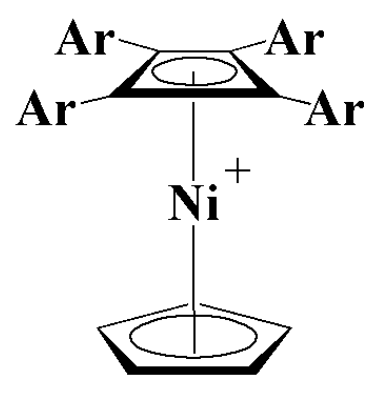

1, $\mathrm{Ar}=\mathrm{Ph}$

1a, $\mathrm{Ar}=o-\mathrm{F}-\mathrm{Ph}$

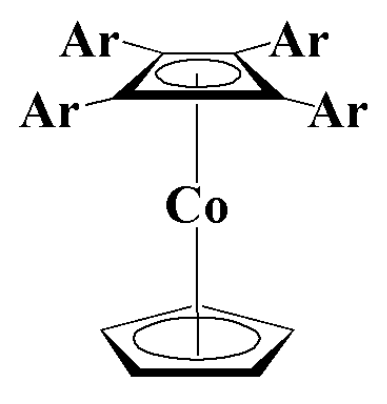

2

Chart 1. Formulas 1, 1a, and 2.

\section{Results}

Properties of the $\left[\mathrm{Ar}_{4} \mathrm{C}_{4} \mathrm{NiCp}\right]^{+}$Sandwich Cation 1. As described in detail in the Supporting Information, the parent 1 was prepared from tolane and $\mathbf{1 a}(\mathrm{X}=o-\mathrm{F}-\mathrm{Ph})$ from 2,2'difluorotolane.

A single-crystal X-ray structure was obtained for the tetrafluoroborate salt of $\mathbf{1}$ and is displayed in Figure 1. Table 1 compares the important structural characteristics, averaged over the approximate local symmetry of the two parts of the molecule, with those calculated for the B3LYP/TZP optimized geometry of the isolated cation 1 (the individual values and their uncertainties are given in Tables S5 and S6). 


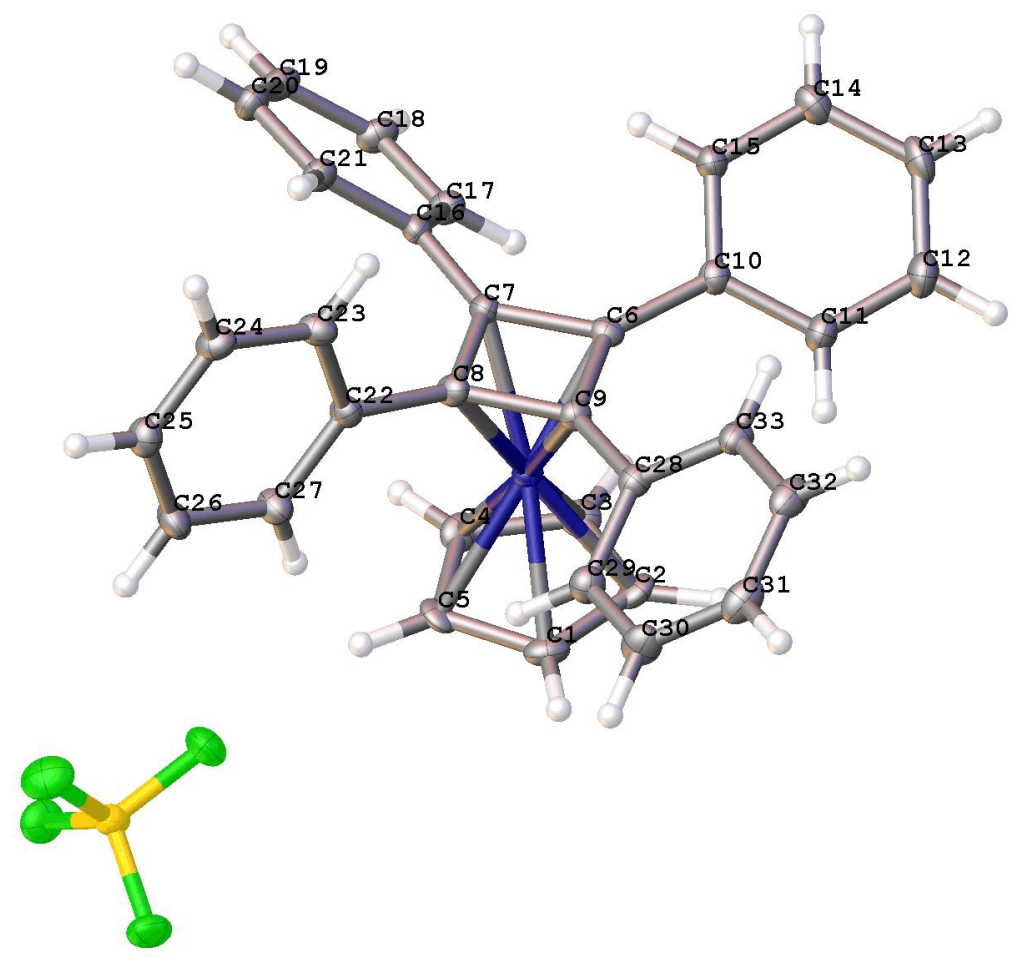

Figure 1. Ortep drawing of the crystal structure of tetrafluoroborate of $\mathbf{1}$.

Table 1. Averaged Structural Characteristics ( $\AA$, deg) of Tetraphenylcyclobutadienecyclopentadienylnickel(II) Tetrafluoroborate (1).

\begin{tabular}{|l|l|l|}
\hline Parameter $^{a}$ & X-ray $\left(\mathrm{C}_{33} \mathrm{H}_{25} \mathrm{BF}_{4} \mathrm{Ni}\right)$ & B3LYP/TZP $\left(\mathrm{C}_{33} \mathrm{H}_{25} \mathrm{Ni}^{+}\right)$ \\
\hline $\mathrm{Ni}-\mathrm{C}(6)(\mathrm{Cb})$ & 2.02 & 2.074 \\
\hline $\mathrm{Ni}-\mathrm{C}(1)\left(\mathrm{Cp}^{-}\right)$ & 2.10 & 2.176 \\
\hline $\mathrm{C}(1)-\mathrm{C}(2)\left(\mathrm{Cp}^{-}\right)$ & 1.426 & 1.430 \\
\hline $\mathrm{C}(6)-\mathrm{C}(7)(\mathrm{Cb})$ & 1.469 & 1.470 \\
\hline $\mathrm{C}(6)-\mathrm{C}(10)(\mathrm{Cb}-\mathrm{Ph})$ & 1.469 & 1.465 \\
\hline $\mathrm{C}(10)-\mathrm{C}(11)(\mathrm{Ph})$ & 1.399 & 1.409 \\
\hline
\end{tabular}




\begin{tabular}{|l|l|l|}
\hline $\mathrm{C}(11)-\mathrm{C}(12)(\mathrm{Ph})$ & 1.394 & 1.393 \\
\hline $\mathrm{C}(12)-\mathrm{C}(13)(\mathrm{Ph})$ & 1.39 & 1.398 \\
\hline $\mathrm{C}(6)-\mathrm{Ni}-\mathrm{C}(7)(\mathrm{Cb})$ & 42.6 & 41.6 \\
\hline $\mathrm{C}(1)-\mathrm{Ni}-\mathrm{C}(2)\left(\mathrm{Cp}^{-}\right)$ & 39.7 & 38.4 \\
\hline $\mathrm{C}(7)-\mathrm{C}(6)-\mathrm{C}(10)$ & 134.4 & 134.2 \\
\hline $\mathrm{C}(8)-\mathrm{C}(6)-\mathrm{C}(10)$ & 171.9 & 170.2 \\
\hline $\mathrm{Ni}-\mathrm{Cp}{ }^{-}$ & 1.714 & 1.804 \\
\hline $\mathrm{Ni}-\mathrm{Cb}$ & 1.732 & 1.795 \\
\hline$\tau(\mathrm{Ph}-\mathrm{Cb})$ & $48.2\left(29.4,39.0,95.4^{b}, 28.9\right)$ & 38.7 \\
\hline
\end{tabular}

${ }^{a} \mathrm{Cb}=$ cyclobutadiene; $\mathrm{Cp}=$ cyclopentadienyl. ${ }^{b}$ Phenyl group in contact with $\mathrm{BF}_{4}^{-}$(two possible C-H $\cdots \cdot \mathrm{F}\left(\delta\right.$-) hydrogen bonds, $\left.\mathrm{d}_{\mathrm{HF}}=2.38,2.51 \AA\right)$

Like the isoelectronic cobalt complex 2, the cations $\mathbf{1}$ and $\mathbf{1 a}$ have a closed-shell singlet ground state and their NMR spectra show no indication that a triplet state is thermally populated. Their electronic absorption spectra of are shown in Figure 2, along with the magnetic circular dichroism (MCD) of $\mathbf{1}$ and the absorption and MCD spectra calculated for this molecule using the TD B3LYP/TZVP method. The calculated excitation energies, intensities, and B terms are listed in Table 2 along with information on the principal excitation amplitudes expressed in terms of single electron excitations from occupied to virtual Kohn-Sham molecular orbitals (MOs). Key MO energies and their shapes are shown in Figure 3 and an MO energy diagram is in Figure 4. We attempted to observe photoluminescence, but were unable to detect any. 


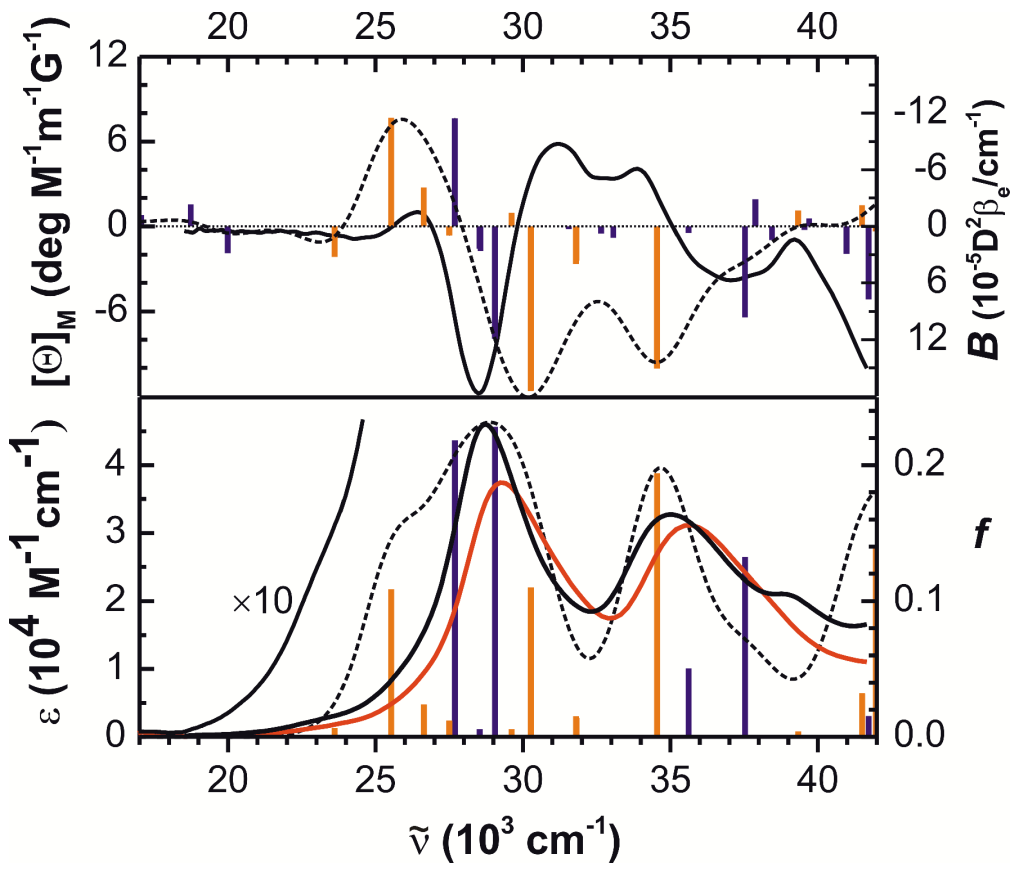

Figure 2. Optical absorption spectra of $\mathbf{1}$ (full black line) and 1a (full red line) and the MCD spectrum of 1 recorded in $\mathrm{CH}_{2} \mathrm{Cl}_{2}$. Bottom: Absorption spectrum of $\mathbf{1}$ overlaid on TDDFT(B3LYP/TZP) calculated oscillator strengths (blue: $z$ polarized; orange: $x, y$ polarized) and simulated spectrum (black dashed line, scaled by a factor of 0.8$)$. Top: MCD spectrum of 1 overlaid on TD-DFT(B3LYP/TZP) calculated B terms (blue: $z$ polarized; orange: $x, y$ polarized) and B3LYP/TZP simulated MCD spectrum (black dashed line, scaled by a factor of 0.7 ).

Table 2. TD-DFT (B3LYP/TZP) calculated vertical transitions in tetraphenylcyclobutadienecyclopentadienylnickel(II) cation $\mathbf{1}$.

\begin{tabular}{|l|l|l|l|l|l|}
\hline No. & $\widetilde{v} / \mathrm{cm}^{-1}$ & $f$ & $B^{a}$ & dominant configurations & polarization \\
\hline 1 & 17060 & 0 & -1.20 & $\mathbf{1} \rightarrow \mathbf{- 2}, \mathbf{2} \rightarrow \mathbf{- 1}$ & $z$ \\
\hline
\end{tabular}




\begin{tabular}{|c|c|c|c|c|c|}
\hline 2 & 18740 & 0 & -2.34 & $2 \rightarrow-1,1 \rightarrow-2$ & $z$ \\
\hline 3 & 19990 & 0 & 2.84 & $1 \rightarrow-1,2 \rightarrow-2$ & $z$ \\
\hline 4 & 23620 & 0.01 & 3.26 & $11 \rightarrow-2,12 \rightarrow-1,7 \rightarrow-1$ & \multirow[b]{2}{*}{$x, y$} \\
\hline 5 & 23620 & 0.01 & 3.24 & $11 \rightarrow-1,12 \rightarrow-2,7 \rightarrow-2$ & \\
\hline 6 & 25530 & 0.11 & -11.52 & $\mathbf{3} \rightarrow \mathbf{- 1}, 13 \rightarrow-1,12 \rightarrow-1$ & \multirow{2}{*}{$x, y$} \\
\hline 7 & 25530 & 0.11 & -11.49 & $\mathbf{3} \rightarrow \mathbf{- 2}, 13 \rightarrow-2,12 \rightarrow-2$ & \\
\hline 8 & 26640 & 0.024 & -4.13 & $3 \rightarrow-2,11 \rightarrow-2,11 \rightarrow-1$ & \multirow{2}{*}{$x, y$} \\
\hline 9 & 26640 & 0.024 & -4.13 & $\mathbf{3} \rightarrow \mathbf{- 1}, 11 \rightarrow-1,11 \rightarrow-2$ & \\
\hline 10 & 27500 & 0.012 & 0.93 & $4 \rightarrow-1,4 \rightarrow-2$ & \multirow{2}{*}{$x, y$} \\
\hline 11 & 27500 & 0.012 & 0.98 & $4 \rightarrow-2,4 \rightarrow-1$ & \\
\hline 12 & 27690 & 0.22 & -11.46 & $2 \rightarrow-2,1 \rightarrow-1,5 \rightarrow-1,6 \rightarrow-2$ & $z$ \\
\hline 13 & 28530 & 0 & 2.37 & $5 \rightarrow-2,6 \rightarrow-1$ & $x, y$ \\
\hline 14 & 28540 & 0.01 & 0.46 & $6 \rightarrow-1,5 \rightarrow-2$ & $z$ \\
\hline 15 & 28570 & 0 & 2.62 & $6 \rightarrow-2,5 \rightarrow-1$ & $x, y$ \\
\hline 16 & 29050 & 0.23 & 11.92 & $6 \rightarrow-2,5 \rightarrow-1,2 \rightarrow-2,1 \rightarrow-1$ & $z$ \\
\hline 17 & 29620 & 0.01 & -1.42 & $8 \rightarrow-1$ & \multirow{2}{*}{$x, y$} \\
\hline 18 & 29620 & 0.01 & -1.41 & $8 \rightarrow-2$ & \\
\hline 19 & 30270 & 0.11 & 17.45 & $7 \rightarrow-2,13 \rightarrow-2,12 \rightarrow-2$ & \multirow{2}{*}{$x, y$} \\
\hline 20 & 30270 & 0.11 & 17.39 & $7 \rightarrow-1,13 \rightarrow-1,12 \rightarrow-1$ & \\
\hline 21 & 31560 & 0 & 0.32 & $9 \rightarrow-1,10 \rightarrow-2$ & $z$ \\
\hline 22 & 31800 & 0.015 & 4.02 & $13 \rightarrow-2,12 \rightarrow-2$ & \multirow[b]{2}{*}{$x, y$} \\
\hline 23 & 31820 & 0.014 & 3.65 & $13 \rightarrow-1,12 \rightarrow-1$ & \\
\hline 24 & 32660 & 0 & 0.77 & $9 \rightarrow-1,10 \rightarrow-2$ & $z$ \\
\hline 25 & 33070 & 0 & 1.22 & $9 \rightarrow-2,10 \rightarrow-1$ & $z$ \\
\hline 26 & 34560 & 0.19 & 15.07 & $1 \rightarrow-3$ & \multirow[b]{2}{*}{$x, y$} \\
\hline 27 & 34560 & 0.19 & 14.88 & $2 \rightarrow-3$ & \\
\hline 28 & 35610 & 0.051 & 0.69 & $14 \rightarrow-1,15 \rightarrow-2,10 \rightarrow-2,9 \rightarrow-1$ & $z$ \\
\hline 29 & 37530 & 0.13 & 9.62 & $9 \rightarrow-2,10 \rightarrow-1$ & $z$ \\
\hline 30 & 37880 & 0 & -2.89 & $15 \rightarrow-2,14 \rightarrow-1$ & $z$ \\
\hline 31 & 38450 & 0 & 1.45 & $15 \rightarrow-1,14 \rightarrow-2$ & $z$ \\
\hline 32 & 39330 & 0 & -1.70 & $1 \rightarrow-4$ & \multirow{2}{*}{$x, y$} \\
\hline 33 & 39340 & 0 & -1.69 & $2 \rightarrow-4$ & \\
\hline 34 & 39550 & 0 & 0.37 & $3 \rightarrow-3$ & $z$ \\
\hline 35 & 39710 & 0 & -0.84 & $4 \rightarrow-3$ & $z$ \\
\hline 36 & 40980 & 0 & 2.93 & $8 \rightarrow-3,2 \rightarrow-6,1 \rightarrow-7$ & $z$ \\
\hline 37 & 41500 & 0.032 & -2.24 & $5 \rightarrow-3,1 \rightarrow-5$ & \multirow[b]{2}{*}{$x, y$} \\
\hline 38 & 41510 & 0.032 & -2.18 & $6 \rightarrow-3,2 \rightarrow-5$ & \\
\hline 39 & 41730 & 0.016 & 7.73 & $7 \rightarrow-3$ & $z$ \\
\hline 40 & 41970 & 0.14 & 0.54 & $1 \rightarrow-5,5 \rightarrow-3$ & \multirow{2}{*}{$x, y$} \\
\hline 41 & 41970 & 0.14 & 0.46 & $2 \rightarrow-5,6 \rightarrow-3$ & \\
\hline 42 & 42360 & 0 & -1.32 & $1 \rightarrow-6,2 \rightarrow-7$ & $z$ \\
\hline 43 & 42430 & 0 & -2.32 & $2 \rightarrow-7,1 \rightarrow-6,2 \rightarrow-6,1 \rightarrow-7$ & $z$ \\
\hline
\end{tabular}




\begin{tabular}{|l|l|l|r|l|l|}
44 & 42790 & 0.084 & -9.62 & $\mathbf{1} \rightarrow \mathbf{- 7}, \mathbf{2} \rightarrow \mathbf{- 6}, 4 \rightarrow-3$ & $z$ \\
\hline 45 & 43630 & 0 & 1.97 & $\mathbf{8} \rightarrow \mathbf{- 3}, 1 \rightarrow-7$ & $z$ \\
\hline 46 & 43850 & 0 & 0.72 & $\mathbf{1} \rightarrow \mathbf{- 9}, \mathbf{2} \rightarrow \mathbf{- 1 0}$ & $z$ \\
\hline 47 & 43970 & 0 & -1.83 & $\mathbf{2} \rightarrow \mathbf{- 1 0}, \mathbf{1} \rightarrow \mathbf{- 9}$ & $z$ \\
\hline 48 & 44190 & 0 & -0.48 & $\mathbf{2 \rightarrow - 9}, \mathbf{1} \rightarrow \mathbf{- 1 0}$ & $z$ \\
\hline 49 & 44380 & 0.014 & -1.19 & $\mathbf{2 \rightarrow - 8}, 6 \rightarrow-3$ & $x, y$ \\
\hline 50 & 44360 & 0.013 & -1.15 & $\mathbf{1 \rightarrow - 8}, 5 \rightarrow-3$ & $z$ \\
\hline 51 & 44590 & 0 & -0.46 & $\mathbf{3 \rightarrow - 4}$ & $z$ \\
\hline 52 & 45100 & 0 & 0.85 & $15 \rightarrow-1,14 \rightarrow-2,12 \rightarrow-3$ & $z$ \\
\hline 53 & 45680 & 0.062 & -1.09 & $\mathbf{1 \rightarrow - 1 0 , 2 \rightarrow - 9}, 3 \rightarrow-5$ & $z$ \\
\hline 54 & 46050 & 0 & 2.09 & $\mathbf{7 \rightarrow - 4}, \mathbf{4} \rightarrow \mathbf{- 5}$ & \\
\hline
\end{tabular}

${ }^{a}$ In units of $10^{-5} \mathrm{D}^{2} \beta_{\mathrm{e}} / \mathrm{cm}^{-1} .{ }^{b}$ Dominant configurations with weight more than $10 \%$ (bold: weight more than $25 \%$ ).

$1=\mathrm{HOMO},-1=\mathrm{LUMO}, 2=\mathrm{HOMO}-1,-2=\mathrm{LUMO}+1$, etc. 

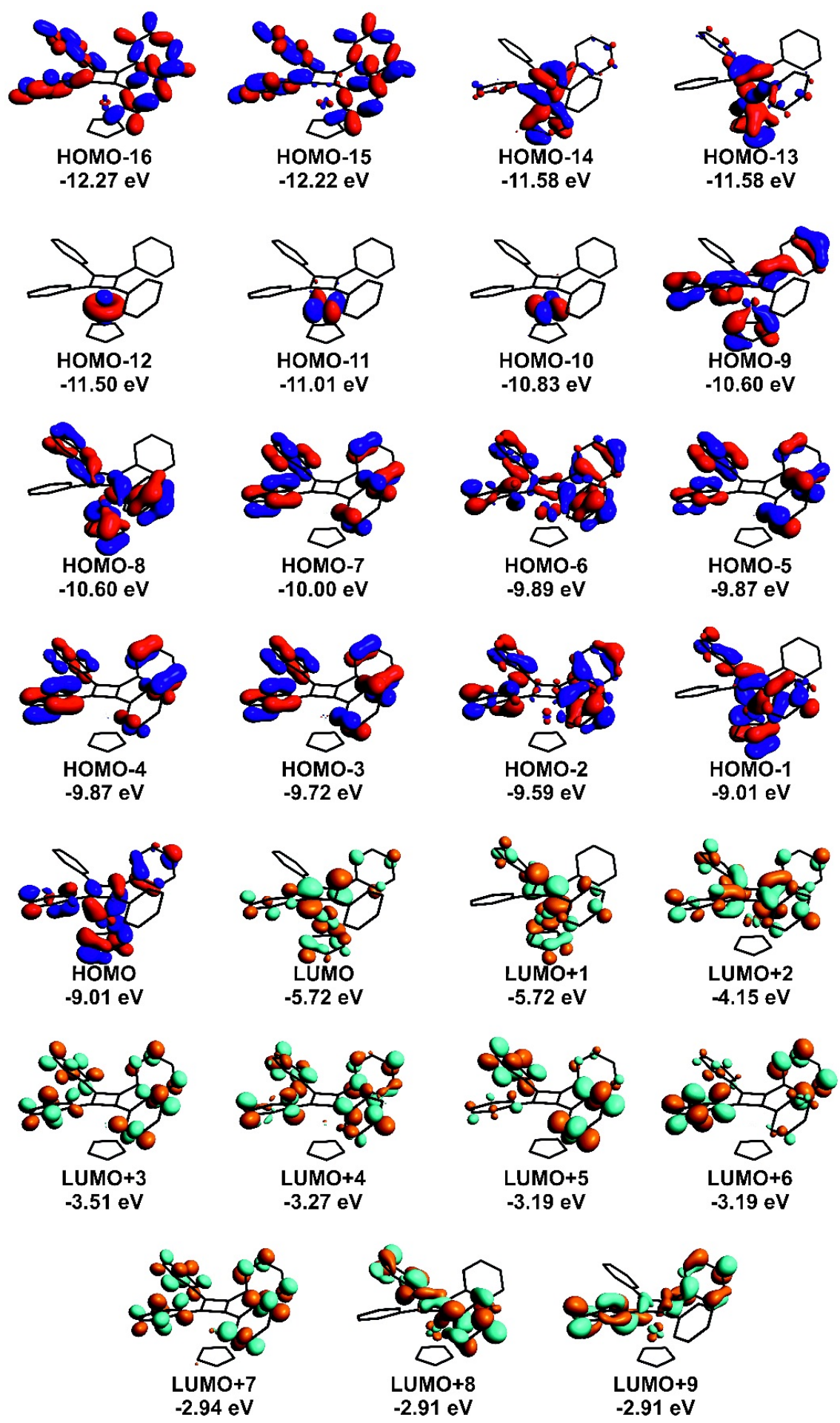

Figure 3. B3LYP/TZP frontier orbitals of $\mathbf{1}$. 


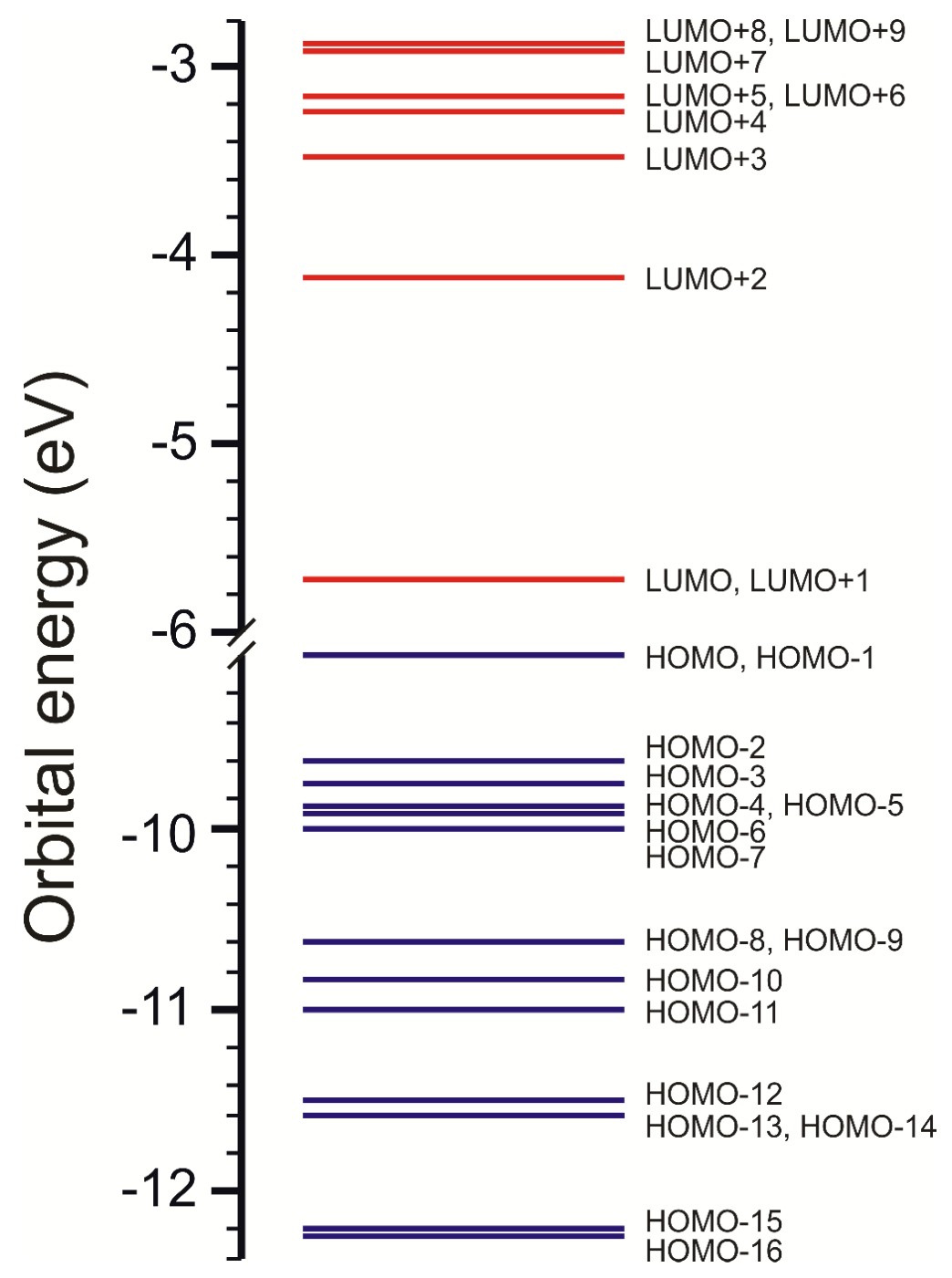

Figure 4. B3LYP/TZP MO energy diagram for 1.

The parent $\mathbf{1}$ is known to be reversibly reduced to a 19 -electron neutral radical at $-740 \mathrm{mV}$ vs NHE in $\mathrm{DMF}^{7}$ and $-730 \mathrm{mV}$ vs. NHE in $\mathrm{CH}_{2} \mathrm{Cl}_{2}{ }^{8}$ Our measurement yielded a similar half-wave reduction potential vs. NHE for the bromide salt in $\mathrm{CH}_{3} \mathrm{CN} / 0.1 \mathrm{M} n-\mathrm{Bu}_{4} \mathrm{~N}^{+} \mathrm{PF}_{6}^{-},-0.765 \mathrm{~V}$. For the bromide of $1 \mathrm{a}$ in $\mathrm{CH}_{3} \mathrm{CN} / 0.1 \mathrm{M} n-\mathrm{Bu}_{4} \mathrm{~N}^{+} \mathrm{PF}_{6}^{-}$, the value was $-0.662 \mathrm{~V}$.

Solvated electrons from pulse radiolysis monitored by absorbance in the visible and near IR 
regions reacted with $\mathbf{1}$ and $\mathbf{1 a}$ in $\mathrm{CH}_{3} \mathrm{CN}$ with a bimolecular rate constant of $1 \times 10^{11} \mathrm{M}^{-1} \mathrm{~s}^{-1}$ to produce the reduced species in $\sim 10 \mathrm{~ns}$, after which the spectra were stable for longer than $1 \mu \mathrm{s}$. Decay of solvated electron absorption was observed in the near infrared while absorbance of the reduced nickel complexes grew, with an absorption peak a little above $700 \mathrm{~nm}$. The peaks of oneelectron reduced forms of both 1 and $1 \mathrm{a}$ have $\varepsilon=6 \times 10^{3} \mathrm{M}^{-1} \mathrm{~cm}^{-1}$ at the maximum. The spectra of the reduced form of both compounds are shown in Figure 5. The radical derived from $\mathbf{1}$ was also produced by chemical reduction with cobaltocene, and yielded the same visible spectrum. Its MCD spectrum is shown in Figure 5. We attempted to calculate the absorption and MCD spectra of the radical by the TD DFT method with the B3LYP functional used successfully for the cation, but obtained no useful results with the B3LYP and the M06 functionals.

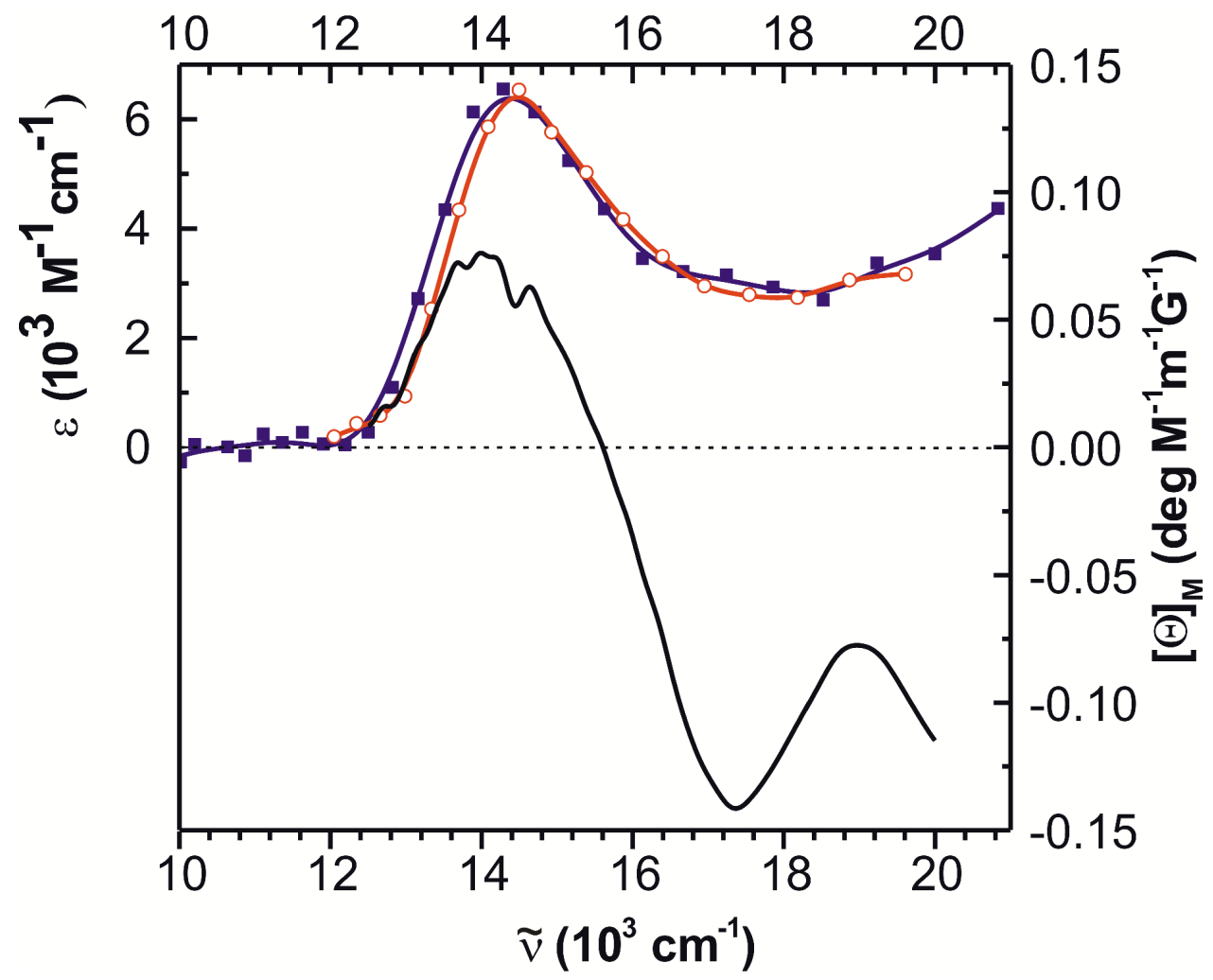

Figure 5. Absorbance at $50 \mathrm{~ns}$ of the singly reduced $\mathbf{1}$ (blue line) and 1a (red line) produced by reaction with solvated electrons, and MCD (black line) of $\mathbf{1}$ singly reduced with cobaltocene, all in acetonitrile solvent. 


\section{Discussion}

Synthesis. The classical synthetic approach works for 1 and its simple derivative 1a, but we find that it is not useful generally. Whereas the availability of only two compounds of this class did not hurt our present efforts to obtain information on its basic electronic characteristics, the absence of a simple general synthetic approach is presently likely to limit the utility of $\mathbf{1}$ in any applications.

Structure. The X-ray structure of the tetrafluoroborate salt of 1 (Figure 1 and Table 1) features a $3.45 \AA$ separation of the two planar rings, with aromatic bond lengths in the fivemembered ring and somewhat longer ones in the four-membered one, with the Ni atom nearly exactly in the middle, and with a propeller-like arrangement of the partially twisted phenyl substituents. It is in remarkably good agreement with the B3LYP/TZP optimized structure of the free ion. The calculated differences between the values of parameters related by the local fourfold and fivefold molecular symmetry are very small, and Table 1 only gives the averages over the calculated values.

The only unexpected aspect of the crystal structure is the inequivalence of the twist angles of the four phenyl rings. While three of them are twisted about $30-40^{\circ}$ out of plane, presumably to accommodate the steric requirements of the ortho hydrogen atoms, the fourth one is twisted nearly perpendicular. In contrast, the B3LYP/TZP optimized structure has all four phenyl groups twisted to almost exactly the same degree, $39^{\circ}$. We attribute the discrepancy to a cation - anion interaction that is missing in the free ion calculation: the ortho and meta hydrogen atoms of the fully twisted phenyl ring that are located on the endo side (towards the Ni atom) are separated by only 2.38 and $2.51 \AA$ from two of the fluorine atoms of a neighboring tetrafluoroborate counterion, respectively, 
and clearly form $\mathrm{C}-\mathrm{H} . . . \mathrm{F}(\delta-)$ hydrogen bonds. We consider it very likely that in solutions containing free ions all four phenyl substituents are twisted to a similar degree, about $30-40^{\circ}$. The helical structure makes the molecule chiral, but NMR spectra show that at room temperature the enantiomers interconvert rapidly.

Molecular Orbitals. In order to discuss the electronic spectrum below, it is useful to describe the nature of the MOs first (Figures 3 and 4). We label $z$ the axis of local symmetry that is common to the two decks of the sandwich complex, and the $x$ axis passes through one of the vertices of the cyclopentadienyl ring. One might expect that the fourfold symmetry of the upper deck will beat against the fivefold symmetry of the lower deck, ruining the symmetry properties of both, but the symmetry properties of the MOs actually are very much those that would be expected for a cylinder. Orbitals without a nodal surface containing the cylindrical axis are nondegenerate and could be called $\sigma$ ( $a$ in the $\pi$ systems of cyclobutadiene and cyclopentadienyl, $d_{z}^{2}$ on the nickel atom), whereas those with one ( $\pi, e$ in cyclobutadiene, $e_{1}$ in cyclopentadienyl, and $d_{x z}, d_{y z}$ on nickel) or two ( $\delta, b$ in cyclobutadiene, $e_{2}$ in cyclopentadienyl, and $d_{x y}, d_{x+y}^{2}{ }^{2}$ on nickel) such nodal surfaces occur in nearly exactly degenerate pairs and hardly mix at all. Owing to the presence of the phenyl substituents, some MOs actually have four such nodal planes and can be called $\varphi$. The $x z$ plane contains one of the vertices of the cyclopentadienyl ring and two vertices of the cyclobutadiene ring. The $y z$ plane contains the other two vertices of the cyclobutadiene ring.

The description of the low-energy virtual orbitals is particularly simple. The lowest energy pair, LUMO and LUMO+1, are of the $\pi$ type, are almost exactly degenerate ("pseudodegenerate") and correspond to the degenerate non-bonding $e_{g}$ orbital pair in the $\pi$ system of square cyclobutadiene, with small contributions elsewhere. As in cyclobutadiene, these orbitals are located 
at quite low energy. They are the terminating orbitals for the first 25 low-energy electronic excitations in $\mathbf{1}$. The following group of higher energy orbitals consists of $\sigma, \pi, \delta$, and $\varphi$ symmetry combinations of the lowest unoccupied orbitals of the four benzene rings, and these only serve as terminating orbitals for excitations that lie above $33000 \mathrm{~cm}^{-1}$.

In contrast, very many of the highest-energy occupied orbitals are important in low-energy electronic excitations. They fall into three groups, $\sigma, \pi$, and $\delta$, plus one orbital of type $\varphi$. Orbitals HOMO-2 and HOMO-12 are of $\sigma$ symmetry. Orbital HOMO-12 is a nearly pure nickel $d_{z}^{2}$ orbital, at quite low energy, and orbital HOMO-2 is the symmetric combination of the symmetric HOMOs of the four benzene substituents. The $a$ orbitals of cyclobutadiene and cyclopentadienyl are even much farther down in energy and do not mix into these highest-energy occupied orbitals significantly. There are four orbital pairs of type $\pi$ : HOMO,HOMO-1; HOMO-4,HOMO-5; HOMO8,HOMO-9; HOMO-13,HOMO-14. Among these, the pair HOMO-4,HOMO-5 stands apart as it only has contributions from the benzene substituents (pairs of antisymmetric benzene HOMOs). In the other three, the $e$ cyclobutadiene orbital, the $d_{x z}$ and $d_{y z}$ nickel orbitals, and the $e_{1}$ cyclopentadienyl orbitals, which are of similar energies, play a role somewhat reminiscent of the one that the three interacting atomic $p$ orbitals play in allyl: in the most stable pair (HOMO-13, HOMO14), they are combined in phase (Ni bonding to both rings), in the middle pair (HOMO-8,HOMO-9), there is a node at the center ( $\mathrm{Ni}$ has a non-bonding relation to both rings), and in the least stable pair (HOMO,HOMO-1) there is a node between $\mathrm{Ni}$ and cyclopentadienyl (but not between $\mathrm{Ni}$ and cyclobutadiene; here, the analogy is imperfect). Orbitals of type $\delta$ are the pure nickel $d$ orbitals HOMO-10 $\left(d_{x y}\right)$ and HOMO-11 $\left(d_{x+y}^{2}{ }^{2}\right)$, and the pure benzene orbitals HOMO-3,HOMO-7, both of which have surprisingly different energies for the two pair members, probably related to the fact that 
it is only the presence of the benzene substituents that permits the existence of two distinct orbitals with two nodal planes in the $z$ axis in cyclobutadiene (in cyclobutadiene itself, the $b_{u}$ representation is not degenerate). Indeed, in orbital HOMO-6, the benzene substituents permit the presence of four such nodal planes, and in our approximate classification this MO has to be labeled $\varphi$.

Electronic Excitation in the $\mathrm{Ph}_{4} \mathrm{C}_{4} \mathbf{N i C} \mathbf{p}^{+}$Cation 1. Assuming that the first four intense transitions overlap to produce the first intense absorption band (Figure 2), the agreement of the observed and the TD B3LYP/TZP calculated electronic absorption spectra is quite remarkable, given the complexity of the structure, and permits us to discuss the two together. The MCD spectrum makes it clear that the first intense absorption band indeed contains at least four transitions.

The spectrum consists of two regions: a weak tail between 18000 and $25000 \mathrm{~cm}^{-1}$, and a series of three intense bands of gradually decreasing intensity at $\sim 29000, \sim 35000$, and $\sim 39000 \mathrm{~cm}^{-1}$. The MCD spectrum agrees with computational predictions to some degree only up to about 30000 $\mathrm{cm}^{-1}$. At higher energies there is no agreement, suggesting that the limit of the utility of the TD DFT computational method has been reached. This is perhaps not surprising, given the complexity of the molecular structure and the high density of overlapping excited states present in the higher energy region. Some of these are non-degenerate and others are pseudo-degenerate. The transition moments of the former tend to lie near the $z$ axis. The latter come in pairs, with the transition moments of one of the pair members $y$ polarized and the other one close to $x$ polarized.

According to the results of calculations (Table 2), the weak and featureless absorption tail at long wavelengths is due to three very weak transitions. The extremely small oscillator strength of the first of them and the associated very small radiative rate constant and long radiative lifetime apparently provide ample opportunities for radiationless deactivation by other processes, and account 
for the absence of observable fluorescence. In these three transitions, electron density is transferred from the Ni $\mathrm{d}_{x z}$ and $\mathrm{d}_{y z}$ orbitals (HOMO,HOMO-1) to the cyclobutadiene $\pi$ non-bonding orbitals (LUMO,LUMO+1), which represent the LUMOs. Such “ $\pi \pi^{*}$ " excitation actually produces four singly excited configurations, which mix to produce a total of four states. The first three are hidden in the weak absorption tail and the fourth one is strongly allowed and is located at a higher energy.

The set of three weakly allowed low-energy states is followed by many additional states produced by excitation into the LUMO orbital pair. The first of these is a pair of weakly allowed pseudo-degenerate states at $23600 \mathrm{~cm}^{-1}$. In these two states excitation from the Ni $d_{x y}$ and $d_{x^{2}+y^{2}}$ orbitals (HOMO-10,HOMO-11) is mixed with excitation from phenyl $\pi$ orbitals (HOMO-7) and the polarization is along $x$ and $y$. The first intense excitations are pseudo-degenerate and are predicted at $25500 \mathrm{~cm}^{-1}$ (oscillator strength $f=0.11$ ). These excitations from phenyl $\pi$ orbitals are polarized in the $x$ and $y$ directions. The next pair of pseudo-degenerate states at $26600 \mathrm{~cm}^{-1}$ carries five times lower intensity. These are primarily excitations from phenyl $\pi$ orbitals. The last pair of pseudodegenerate states at $27500 \mathrm{~cm}^{-1}$ is even twice weaker. These excitations are again from phenyl $\pi$ orbitals.

The pseudo-degenerate transitions are followed by an intense $(f=0.22)$ transition at 27700 $\mathrm{cm}^{-1}$ polarized in the $z$ direction. Slightly above are three transitions at about $28500 \mathrm{~cm}^{-1}$. Two of these are a pseudo-degenerate pair polarized in $x, y$ direction and one is polarized in $z$ direction. All are excitations from phenyl $\pi$ orbitals. At $29100 \mathrm{~cm}^{-1}$ an intense $(f=0.23)$ transition of phenyl $\pi$ to LUMO is predicted, polarized in the $z$ direction. It is followed by two sets of pseudo-degenerate transitions $\left(29600,30300 \mathrm{~cm}^{-1}\right)$ of small $(f=0.005)$ and medium $(f=0.11)$ intensity. The first set has a component of excitation from $\mathrm{Ni} d_{z}^{2}$ orbital to LUMO and the second set is again of phenyl $\pi$ 
to LUMO character. These transitions are polarized in the $x$ and $y$ directions.

The character of the next five transitions with small to medium intensity is similar. The transitions with small intensity are of phenyl $\pi$ to LUMO nature, and the medium intensity transitions have Ni $d$ orbital to LUMO character. The next pseudo-degenerate pair of transitions is the first one in which electrons are excited into a higher unoccupied orbital, located on the phenyl groups. The intensity is high $(f=0.19)$ and the polarization is in $x$ and $y$ directions. It is followed by a medium intensity transition at $35600 \mathrm{~cm}^{-1}$ polarized in the $z$ direction (excitation from a $\mathrm{Ni} d$ orbital to LUMO) and by the next intense transition at $37500 \mathrm{~cm}^{-1}(f=0.13)$ of partly cyclopentadienyl-tocyclobutadiene character.

In the region between 37800 to $46000 \mathrm{~cm}^{-1}$ seven states of very small and small intensity are predicted, plus a pseudo-degenerate pair of transitions at $42000 \mathrm{~cm}^{-1}$ with high intensity $(f=$ 0.14 ) with HOMOs to phenyl $\pi^{*}$ character, polarized in $x$ and $y$ directions.

Electronic Excitation in the $\mathbf{P h}_{4} \mathbf{C}_{4} \mathbf{N i C p}$ Radical. The one-electron reduction of $\mathbf{1}$ at $-0.77 \mathrm{~V}$ against NHE places an electron into the nearly degenerate -1,-2 LUMO pair, almost entirely localized in the cyclobutadiene ring (Figure 3), and is expected to cause a pseudo-Jahn-Teller distortion from its square geometry. The introduction of four fluorine substituents upon going from 1 to 1 a makes the reduction potential more positive by $\sim 0.1 \mathrm{~V}$. The increase can be attributed to a small stabilization of the lowest unoccupied molecular -1,-2 LUMO pair, which has only very small coefficients on the phenyl substituents.

Since we were unable to obtain any meaningful results from TD DFT calculations on the neutral radical produced by one-electron reduction of $\mathbf{1}$, the following discussion of its spectrum is strictly qualitative. The intense low-energy electronic transition observed in the visible region 
(Figure 5) is attributed to a transition of the added electron from its singly occupied molecular orbital pair $-1,-2$, SOMO, to the orbital -3 . This excitation is nearly exclusively localized in the tetraphenylcyclobutadiene portion of the molecule. It corresponds to the allowed $e_{g} \rightarrow b_{u}$ excitation in the parent cyclobutadiene radical cation, but in the latter it would mix with a nearly isoenergetic $a_{2 u} \rightarrow e_{g}$ excitation, which is absent presently. The small effect of tetrafluoro substitution, apparent from a comparison of the absorption spectra of the reduced forms of $\mathbf{1}$ and 1a (Figure 2), is attributable to the very similar stabilization of the SOMO and the LUMO by the added fluorine substituents.

In the limit of perfect degeneracy, this type of excitation in cyclobutadiene radical cation corresponds to a transition with a negative $A$ term, since a left-handed circularly polarized photon promotes an electron from an orbital in which it has a negative component of the orbital magnetic dipole moment along the $z$ axis into a non-degenerate orbital where it has a zero orbital magnetic moment. ${ }^{9}$ In this regard, the situation is similar to that in cyclobutadiene dianion, whose symmetrical tetrasilylated derivative has been prepared recently and indeed exhibited the expected negative $A$ term. ${ }^{10}$ The lower symmetry of the $\mathrm{Ph}_{4} \mathrm{C}_{4} \mathrm{NiCp}$ radical causes us to expect a pseudo- $A$ term, experimentally indistinguishable from an $A$ term, and this is indeed observed (Figure 2), in support of the proposed assignment. A study of temperature dependence should also reveal a presence of a pseudo- $C$ term, but could not be performed with the equipment on hand. We propose that the observation of a negative $A$ term supports the assignment sufficiently.

Experimental Procedures. All experimental details are provided in the Supporting Information.

Computations. Calculations were performed by the DFT-B3LYP method using the program 
ADF, version 2013. ${ }^{11}$ The internally stored Slater-type basis set ${ }^{12}$ TZP was used for all calculations (geometry optimization, excitation spectra, MCD). For details, see Supporting Information.

Supporting Information Available. Experimental details for measurements and synthesis. Computational methods, optimized geometries, and details of X-ray diffraction structure determination. This material is available free of charge via the Internet at http://pubs.acs.org.

Acknowledgment. This material is based upon work supported by the National Science Foundation under Grant No. CHE-1265922. We are grateful to Mr. Justin M. Ray, Mr. David J. Koditek, and Mr. Kamal Gala for technical assistance. Work in Prague was supported by the Institute of Organic Chemistry and Biochemistry (RVO:61388963) and the Czech Science Foundation (GBP208/12/G016). 


\section{References}

1. Efraty, A. Cyclobutadienemetal Complexes. Chem Rev. 1977, 77, 691-744.

2. Harrison, R. M.; Brotin, T.; Noll, B. C.; Michl, J. Toward a Square-Grid Polymer: Synthesis and Structure of Pedestal-Mounted Tetragonal Star Connectors, $\mathrm{C}_{4} \mathrm{R}_{4}-\mathrm{Co}-\mathrm{C}_{5} \mathrm{Y}_{5}$. Organometallics 1997, 16, 3401-3412.

3. Brotin, T.; Pospíšil, L.; Fiedler, J.; King, B. T.; Michl, J. Toward a Square Grid Polymer: Electrochemistry of Tentacled Tetragonal Star Connectors, $\mathrm{C}_{4} \mathrm{R}_{4}-\mathrm{Co}_{-} \mathrm{C}_{5}(\mathrm{HgY})_{5}$, on Mercury. J. Phys. Chem. B 1998, 102, 10062-10070.

4. Kottas, G. S.; Brotin, T.; Schwab, P. F. H.; Gala, K.; Havlas, Z.; Kirby, J. P.; Miller, J. R.; Michl, J. Tetraarylcyclobutadienecyclopentadienylcobalt Complexes: Synthesis, Electronic Spectra, Magnetic Circular Dichroism, Linear Dichroism and TD DFT Calculations. Organometallics 2014, 33, 3251-3264.

5. Maitlis, P. M.; Efraty, A.; Games, M. L. Cyclobutadiene-Metal Complexes. IV. ${ }^{1}$ ( $\pi$-Cyclopentadienyl) ( $\pi$-tetraphenylcyclobutadiene) palladium Halides and Related Complexes. J. Am. Chem. Soc., 1965, 87, 719-724.

6. Pollock, D. F.; Maitlis, P. M. Cyclobutadiene-metal Complexes XII. Syntheses and Some Reactions of para-substituted-(tetraphenylcyclobutadiene)palladium halides. J. Organomet. Chem. 1971, 26, 407-415.

7. Herberich, G. E.; Klein, W.; Kölle, U.; Spiliotis, D. Elektronenüberschuß-Komplexe von Cobalt und Nickel mit Cyclobutadien-Liganden: Synthese, Cyclovoltammetrie und Reaktivität. Chem. Ber. 1992, 125, 1589-1595.

8. Kölle, U.; Ting-Zhen, D.; Keller, H.; Ramakrishna, B. L.; Raabe, E.; Krüger, C.; Raabe, G.; 
Fleischhauer, J. Reduktion von(Cyclobutadien)(cyclopentadienyl)- und (1,5-Cyclooctadien)(cyclopentadienyl)nickel-Kationen. Chem. Ber. 1990, 123, 227-235.

9. Michl, J. MCD of Cyclic $\pi$-Electron Systems. I. Algebraic Solution of the Perimeter Model for the A and B Terms of High-Symmetry Systems with a (4N+2)-Electron [n]Annulene Perimeter. J. Am. Chem. Soc. 1978, 100, 6801-6811.

10. Ishii, K.; Kobayashi, N.; Matsuo, T.; Tanaka, M.; Sekiguchi, A. Observation of the Predicted Negative Faraday A MCD Term in a Cyclobutadiene Dianion J. Am. Chem. Soc. 2001, 123, 5356-5357.

11. Baerends, E. J.; Ziegler, T.; Autschbach, J.; Bashford, D.; Bérces, A.; Bickelhaupt, F. M.; Bo, C.; Boerrigter, P. M.; Cavallo, L.; Chong, D. P. et al. ADF2013, SCM, Theoretical Chemistry, Vrije Universiteit, Amsterdam, The Netherlands, http://www.scm.com.

12. van Lenthe, E.; Baerends, E. J. Optimized Slater-type basis sets for the elements 1-118. J. Comput. Chem. 2003, 24, 1142-1156. 
TOC Graphic

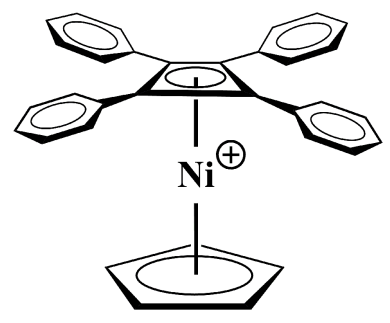

